\title{
Answer to the Letter to the Editor of Akbari KK et al. concerning "The short-term impact of COVID-19 pandemic on spine surgeons: a cross-sectional global study" by Khattab MF, et al. (Eur Spine J; [2020]: doi:10.1007/s00586-020-06517-1)
}

\author{
Anouar Bourghli ${ }^{1} \cdot$ Mohamed Fawzy Khattab $^{2} \cdot$ Tareq M. A. Kannan $^{3} \cdot$ Ibrahim Obeid $^{4}$
}

Received: 5 July 2020 / Accepted: 15 July 2020 / Published online: 29 July 2020

(c) Springer-Verlag GmbH Germany, part of Springer Nature 2020

\section{Dear Editor,}

The questions raised in the letter concerning the study by Khattab et al. [1] are important, and we completely agree with the authors.

With regard to the method of sampling, it is true that it was not a snowball sampling method as in the classical definition of "chain method" like it was defined by the authors; the term was nevertheless employed, like in different recent studies in the literature [2,3], because the questionnaire was initially sent by the initiators of the study to a certain number of spine surgeons in different countries who are either friends, or previous colleagues or involved in the spinal society of the country, and then they actively helped in the distribution of the questionnaire, by emails or internet applications, to the many other surgeons across the respective countries and globally across the globe. Given the studied subject with the context of COVID-19, it would have been difficult to apply the rules of probability [4] for the sampling and as the study concerned only specifically spine surgeons, target community representation could be guaranteed.

The fact that 780 "agreed" on filling the questionnaire was a sentence that may have been misunderstood by the readers, as it may have been sloppily worded. In fact, we did

Ibrahim Obeid

ibrahim.obeid@gmail.com

1 Orthopedic and Spinal Surgery Department, Kingdom Hospital, Riyadh, Saudi Arabia

2 Orthopedic Surgery Department, Faculty of Medicine, Ain-Shams University, Cairo, Egypt

3 Spine Unit, Jordan University Hospital, University of Jordan, Amman, Jordan

4 Spine Surgery Unit, ELSAN Group Jean Villar Private Hospital, 33000 Bruges-Bordeaux, France not mean that there was a pre-agreement as it is true that preagreed convenience sampling raises concerns about the final outcome and may bias the study; it was rather another way to state what was initially formulated in the method and the fact that around $26 \%$ of the surgeons took part in the study.

Reaching out for as many surgeons across the globe in the different regions was a difficult task, with a very limited time frame; this is why there was a discrepancy in the sample sizes across the continents with some regions that are more represented than others. But with regard to South Asia region, it was not specifically named as it was part of the far-East and mid-Asia region.

We appreciate the letter above, and we hope the answers will help to understand the difficulties encountered when making a global scale study during a planetary crisis such as the COVID-19 pandemic.

\section{Compliance with ethical standards}

Conflict of interest The authors declare that they have no confict ofinterest.

\section{References}

1. Khattab MF, Kannan TMA, Morsi A, Al-Sabbagh Q, Hadidi F, Al-Sabbagh MQ, Taha MM, Bourghl A, Obeid I (2020) The shortterm impact of COVID-19 pandemic on spine surgeons: a crosssectional global study. Eur Spine J. https://doi.org/10.1007/s0058 6-020-06517-1

2. Thomaier L, Teoh D, Jewett P, Beckwith H, Parsons H, Yuan J, Blaes AH, Lou E, Hui JYC, Vogel RI (2020) Emotional health concerns of oncology physicians in the United States: fallout during the COVID-19 pandemic. medRxiv. https://doi. org/10.1101/2020.06.11.20128702 
3. Perez-Fuentes MDC, Molero Jurado MDM, Martos Martinez A, Gazquez Linares JJ (2020) Threat of COVID-19 and emotional state during quarantine: positive and negative affect as mediators in a cross-sectional study of the Spanish population. PLoS ONE 15:e0235305. https://doi.org/10.1371/journal.pone.0235305

4. Ebert JF, Huibers L, Christensen B, Christensen MB (2018) Paper- or web-based questionnaire invitations as a method for data collection: cross-sectional comparative study of differences in response rate, completeness of data, and financial cost. J Med Internet Res 20:e24. https://doi.org/10.2196/jmir.8353

Publisher's Note Springer Nature remains neutral with regard to jurisdictional claims in published maps and institutional affiliations. 